\title{
A novel technique for extensor mechanism repair
}

\section{Abstract}

Innovations in orthopaedic surgery have led to advancements in repair techniques for extensor mechanism tendon tears. The most popular method has been the three bone tunnel technique. This involves a locking suture placed into the substance of the torn extensor tendon and passing this through three vertical tunnels through the patella bone. The Zip Loop Toggle Loc Device (Biomet Sports Medicine Warsaw, Indiana) has been used in multiple applications to include cruciate ligament reconstruction, acute $\mathrm{AC}$ joint dislocation, distal biceps tendon repair, and ankle syndesmotic injuries. We propose the use of the ZipLoopToggleLocDevice in a novel technique for the repair of extensor mechanism tears. To our knowledge, the use of the Zip Loop Device in extensor mechanism repair has not been described.
Volume 2 Issue 6 - 2015

\author{
Juluru P Rao,Alvaro Cabezas, Jacob A \\ Spencer, Edward Mastromonaco \\ Jersey City Medical Center, USA
}

Correspondence: Jacob A Spencer, Jersey City Medical Center, 355 Grand Street, New Jersey, USA, Tel 9092622475, Email jspencerdo@gmail.com

Received: April 22, 2015 | Published: June 19, 2015

Keywords: Extensor mechanism, Zip Loop Toggle Loc Device

\section{Introduction}

Extensor mechanism injuries are relatively infrequent but can have a debilitating outcome if not treated in a timely fashion. Historically, injuries to the quadriceps or patellar tendon incorporated the use of an augment in the form of cerclage wire, followed by prolonged immobilization. ${ }^{1}$ Advancement in techniques, augment materials and early functional rehabilitation has improved the quality of mobilization in this patient population.

\section{Case report}

GC, a 38-year-old male, presented to the emergency room with complaints of right knee pain. The patient admitted to landing awkwardly one day ago while playing basketball and heard a popping sound that he localized to the right knee. He had immediate pain and swelling and was unable to bear weight. On exam, he had a palpable defect in his patellar tendon and was unable to straighten his leg against gravity. He was evaluated with x-rays (Figures 1A \& 1B), which demonstrated a high riding patella. After discussing the treatment options and risks, benefits and complications of surgery, he was taken to the operating room where his patella tendon was repaired.
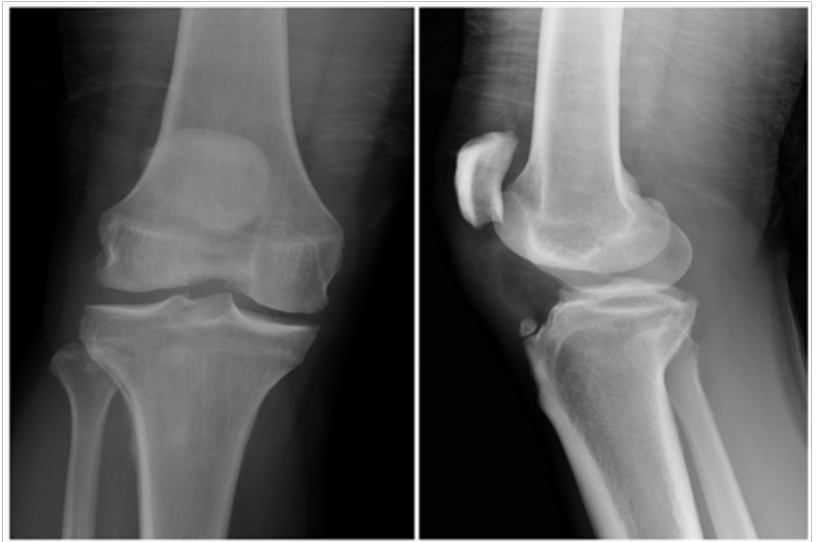

Figure IA \& IB Initial injury anteroposterior and lateral radiographs demonstrating patella alta.

\section{Technique}

\section{Incision}

The patient was positioned supine on the operating room table and his right leg was prepped and draped in a sterile fashion. A midline longitudinal incision was made from approximately $2 \mathrm{~cm}$ proximal to the superior pole of the patella, extending distally to approximately $2 \mathrm{~cm}$ proximal and $1 \mathrm{~cm}$ medial to the tibial tuberosity. Careful attention was paid to preserve the paratenon to facilitate closure after repair of the tendon to aid in healing and the prevention of adhesions. The ruptured extensor mechanism was then exposed (Figure 2).

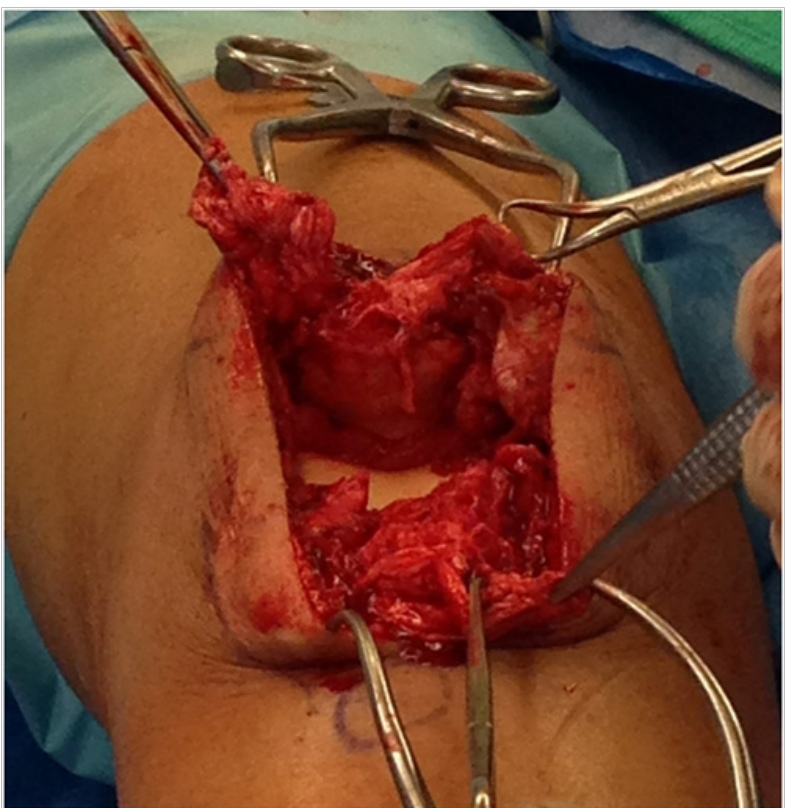

Figure 2 Intraoperative photograph of exposed patellar tendon demonstrating rupture with frayed ends.

\section{Tendon preparation}

The disrupted extensor tendon and retinaculum were identified. Macerated tendon edges were sharply debrided. The Zip Loop sutures 
(\#2 Max Braid) with attached tapered needles were attached to the end of the torn patellar tendon utilizing a whipstitch technique (Figure 3).

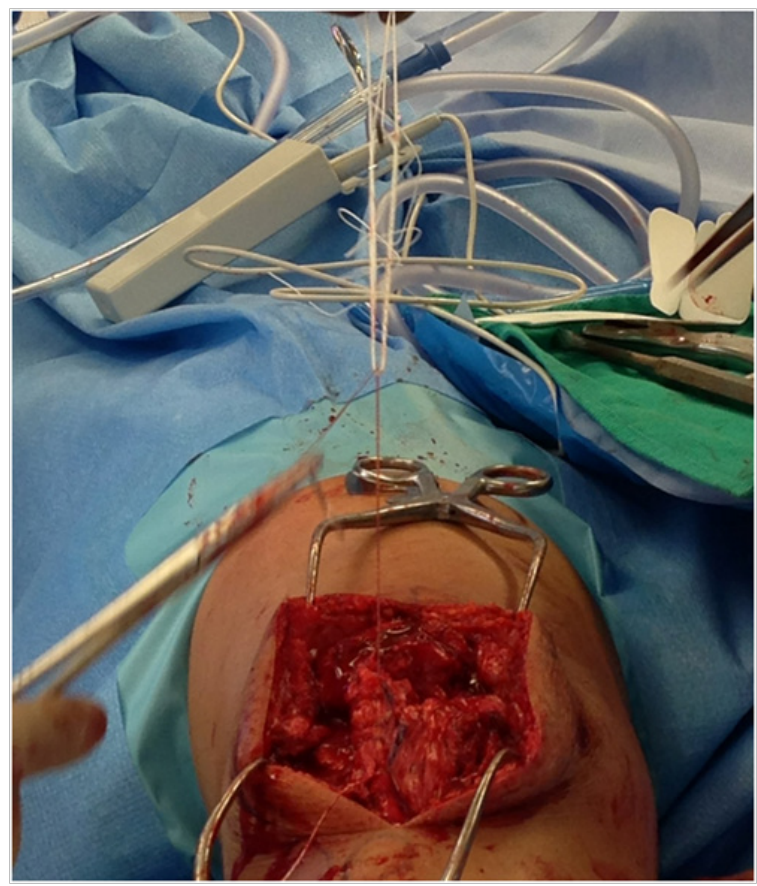

Figure 3 Intraoperative photograph demonstrating ZipLoop sutures "whipstitched" into the patellar tendon ends.

\section{Preparation of tunnels}

The origin of the patellar tendon was identified and debrided to bleeding bone. After identifying the medial and lateral borders of the patella, the patella was visually divided into thirds and the starting points for the guide wires were marked with a bovie at the one-third and two-thirds margin to ensure appropriate spacing between tunnels. Under fluoroscopic guidance, two $2.4 \mathrm{~mm}$ guide wires were inserted through the patella, starting from the anterior edge of the inferior poleto the posterior aspect of the superior pole in a parallel fashion. The articular surface of the patella was palpated to ensure there was no penetration. The tips of the guide wires were palpate with a freer, then advanced through the quadriceps tendon and secured with kocher clamps (Figure 4). As recommended by the manufacturer for passage of the Toggle Loc device, a $4.5 \mathrm{~mm}$ cannulated drill was carefully passed through bone.

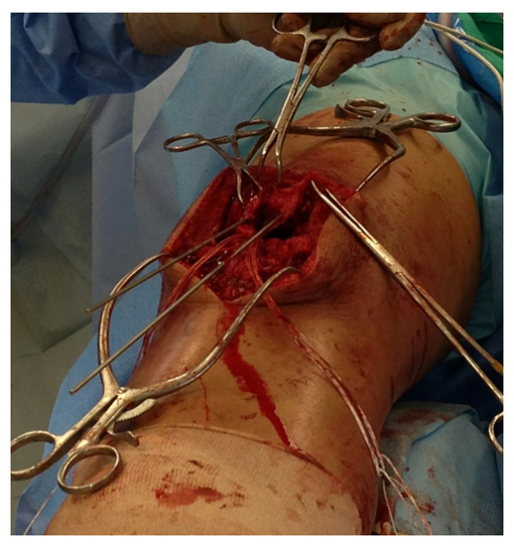

Figure 4 Intraoperative photograph demonstrating the passage of two $2.4 \mathrm{~mm}$ guide wires through the patella in preparation for drilling with a $4.5 \mathrm{~mm}$ canulated drill and then passage of the ToggleLoc sutures.

\section{Passing of the toggle loc suture device}

Abeath pin and passing suture (\#1 Vicryl) for the Toggle Loc fixation device were carefully passed through the $4.5 \mathrm{~mm}$ tunnels in the patella (Figure 5). The Toggle Loc device was then pushed through using the Toggle Loc pusher and flipped with an obturator. The sutures were then gently tensioned to ensure the device was secure. With the knee in full extension and the patella centered in its anatomic position within the trochlea, the zip strand of the Zip Loop device was pulled to close the gap between the repair site and superior pole of the patella. The strands were individually tensioned to remove any excess slack, while maintaining the patella's position. The knee was then ranged and assessed for gapping of the repair, tracking of the patella and tension of the extensor mechanism during flexion.

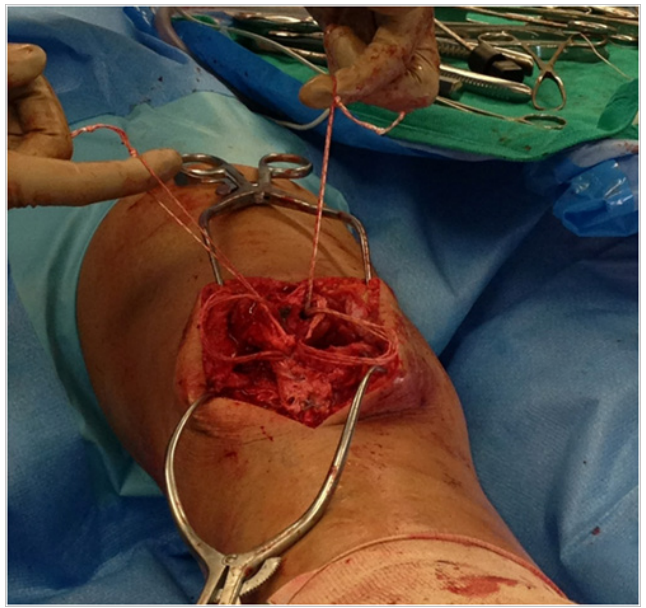

Figure 5 Intraoperative photograph demonstrating the passage of the ZipLoop sutures through the $4.5 \mathrm{~mm}$ tunnels in the patella.

\section{Closure and rehab}

The wound was irrigated followed by a layered closure. The retinaculum was repaired with \#1Vicryl and \#0 Vicryl was used to augment portions of the patellar tendon repair near the origin to debulk and reinforce the repair site. \#0 Vicryl was then used to close the paratenon and then the deep fascia. 2-0 Vicryl was then used for subcutaneous fascia followed by staples for the skin. Early flexion was determined Intraoperatively by the quality of tendon tissue and the repair. The knee could be flexed up to 90 degrees without any gaping of the repair. Sterile dressing was applied and the knee was placed in a knee immobilizer, locked in extension. Radiographs done immediately postoperatively revealed the Toggle Loc deployed and securely in place with good anatomic alignment of the patella within the trochlear groove (Figures 6A \& 6B).

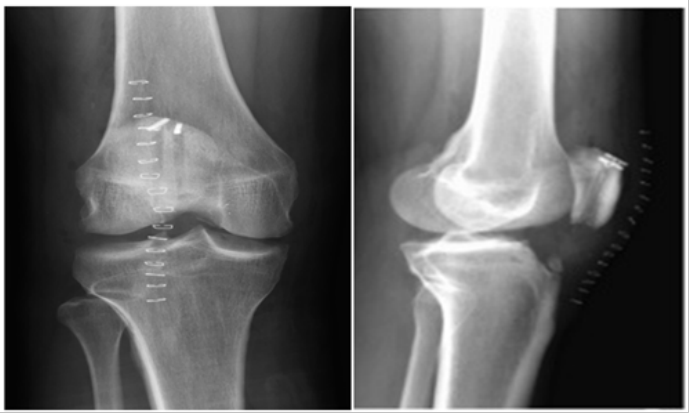

Figure 6A \& 6B Postoperative anteroposterior and lateral radiographs of the knee demonstrating successful ToggleLoc deployment and restoration of the patella's orientation. 
The patient was made full weight bearing with crutch assistance immediately after surgery. Progressive active flexion was initiated at approximately two weeks, after the wound had healed. Active assisted range of motion was implemented at four weeks, with the goal of 90 degrees by six weeks and full ROM by 12 weeks. A strengthening program of isometric straight leg raises was started at six weeks. Radiographs performed at 11 months postoperatively demonstrated no displacement of the ToggleLoc or loss of fixation and the patella remained in its original postoperative position within the trochlear groove (Figures 7A \& 7B).
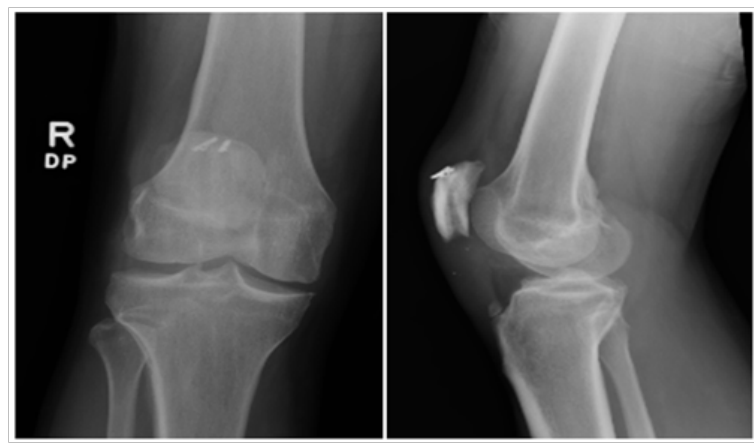

Figure 7A \& 7B II-month postoperative follow-up anteroposterior and lateral radiographs of the knee demonstrating no change in position of the patella or ToggleLoc devices.

The patient was last seen at 13 months postoperatively and demonstrated symmetric quadriceps size, mild tenderness at the superior-medial pole of the patella, $0-125$ o of active knee ROM, 5/5 quadriceps strength and an intact straight leg raise with $4 / 5$ strength to resisted straight leg raise. The patient subsequently received an MRI at 13 months postoperatively as part of a work-up to re-evaluate the repair after the patient reported a fall. The MRI demonstrated an intact repair with no migration of the Toggle Locs or change in position of the patella relative to the joint line when compared to prior radiographs (Figures $8 \mathrm{~A} \& 8 \mathrm{~B}$ ).
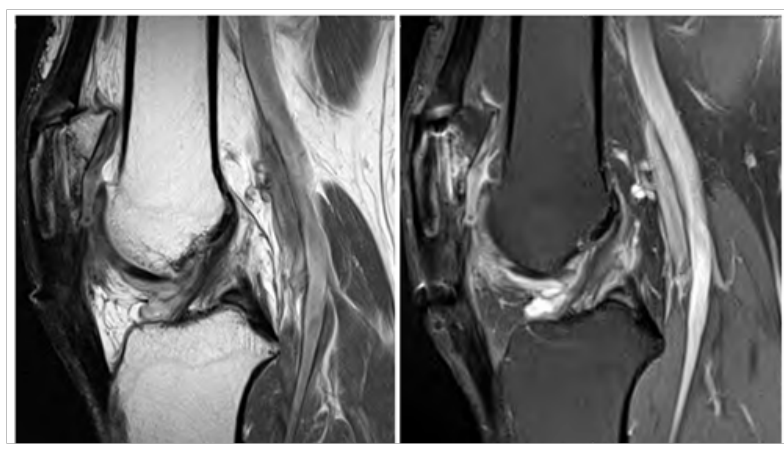

Figure 8A \& 8B I3-month follow-up MRI of the knee demonstrating no change in position of the patella or ToggleLoc devices.

\section{Discussion}

Rupture of the extensor mechanism is a debilitating injury in any patient, but especially devastating in the athletic population. Patellar tendon rupture occurs from a violent contraction of the quadriceps muscle against a statically loaded, flexed knee under the force of the patient's body weight. This is infrequently observed in football, basketball and soccer players when the player is landing or starting a jumping movement. Rupture is thought to be the end result of a patellar tendinosis process due to recurrent micro trauma. It has also been observed in falls from heights. Patellar tendon ruptures occur more frequently in the younger population, under the age of forty, whereas quadriceps tendon ruptures are more common in patients older than forty. ${ }^{2}$

The management of these injuries is continuously evolving. The treatment option ultimately depends on the location of the rupture and whether it is complete or partial. For the purpose of this paper, we focused on complete ruptures at the most frequent location, near the patellar tendon origin at the anterior aspect of the inferior pole of the patella, necessitating bone-to-tendon fixation. ${ }^{3}$ Currently, the three-bone tunnel technique for extensor mechanism repair is widely accepted. While this technique has been very effective, it has the following disadvantages: Retrieval of the suture once passed through the patella can be quite difficult and time consuming and may also require cutting or dissecting into healthy extensor tendon tissue to identify the suture; Three bone tunnels need to be drilled, potentially weakening the bone and prolonging operative time; The strength, location and uniformity of the knot at the proximal pole of the patella can be quite variable and further time consuming. ${ }^{4}$

With the ZipLoopToggleLocDevice, the disadvantages of the conventional three-bone-tunnel technique are resolved. The need to visualize the sutures proximally is not necessary, thus sparing dissection into intact extensor tendon. The placement of just two tunnels can decrease operative time and weakening of the bone by a third tunnel. There is no need for knot tying, as the Toggle Loc provides a uniform and secure fixation. Lastly, this technique can also be applied to quadriceps tendon tears, which the author has used successfully. A potential disadvantage of this technique is failure of the Toggle loc device. The toggle could potentially slide back into the drilled tunnel if it disengages itself from the cortex. While we did not experience this complication, we do recognize it's potential. With careful attention to technique and proper tensioning, this complication can be minimized. Alternatively, in the instance where the patellar tendon is attenuated or there is tissue loss, a \#5 Fiber wire may be passed transversely across the quadriceps tendon near the superior pole of the patella and tied to a second \#5 Fiber wire passed transversely across the patellar tendon near the tibial tuberosity to ensure the height of the patellar tendon is within normal limits so to avoid over tensioning and subsequent patella baja.

The evolution of extensor tendon repair of the knee has led to the current advancements today. Further innovative ways to optimize this surgical procedure can potentially benefit patient outcomes.

\section{References}

1. Marder RA, Timmerman LA. Primary Repair of Patellar Tendon Rupture Without Augmentation. Am J Sports Med. 1999;27(3):304--307.

2. Siwek CW, Rao JP. Ruptures of the Extensor Mechanism of the Knee Joint. J Bone Joint Surg Am. 1981;63(6):932-937.

3. Matava MJ. Patellar Tendon Ruptures. J Am Acad Orthop Surg. 1996;4(6):287-296.

4. Ilan DI, Tejwani N, Keschner M, et al. Quadriceps Tendon Rupture. J Am Orthop Surg. 2003;11(3):192-200. 